\section{Re: the Locker Puzzle}

\section{ERIC Grunwald}

The Mathematical Intelligencer encourages comments about the material in this issue. Letters to the editor should be sent to either of the editors-in-chief, Chandler Davis or Marjorie Senechal.
乙7 aving been astonished by the Locker Puzzle and its winning strategy (Curtin and Warschauer, Mathematical Intelligencer 28 (2006), no. 1), I was delighted to read A. S. Landsberg's letter (Mathematical Intelligencer 31 (2009), no. 2) with its charming variant on the original problem. I would like to point out that Curtin and Warschauer's strategy is so impressive that, amazingly, a third person can be introduced into Landsberg's game without reducing the overall probability of success.

Thus there are three curtains, behind which are hidden a car, a car key, and a GPS system. Three people, the carmaster, the key-master, and the GPS-master, have two attempts each to find their designated object. After each one has tried, the curtains are pulled closed again, and once they have started looking, there is no communication between them. The strategy ensures a probability of $2 / 3$ that all three people find their own object.

Mathematical Capital Limited

187 Sheen Lane

London SW14 8LE

UK

e-mail: ericgrunwald@aol.com 